Владикавказский математический журнал 2017, Том 19, Выпуск 1, С. 30-40

УДК 517.518.13+517.983.23

\title{
О КОМБИНАЦИЯХ ДИФФЕОМОРФНЫХ СДВИГОВ ОКРУЖНОСТИ И НЕКОТОРЫХ ОДНОМЕРНЫХ ИНТЕГРАЛЬНЫХ ОПЕРАТОРОВ
}

\section{С. Б. Климентов}

В работе изучаются суперпозиции диффеоморфизмов единичной окружности и сингулярных интегральных операторов на этой окружности. Установлено свойство таких суперпозиций, аналогичное свойству бесселевых потенциалов. Приводится пример, показывающий, что полученный результат, вообе говоря, не улучшаем.

Ключевые слова: сдвиг контура, сингулярный интегральный оператор.

\section{1. Введение. Формулировка результатов}

Обозначим через $D=\{z:|z|<1\}$ единичный круг комплексной $z$-плоскости $E$, $z=x+i y, i^{2}=-1 ; \Gamma=\partial D-$ граница круга $D ; \bar{D}=D \cup \Gamma$.

В работе используется банахово пространство $C^{k, \alpha}(\Gamma)$ комплекснозначных функций, имеющих на $\Gamma k$ производных, где $k \geqslant 1$ - целое число, причем $k$-е производные удовлетворяют условию Гёльдера с показателем $\alpha, 0 \leqslant \alpha \leqslant 1$. В этом пространстве предполагается заданной стандартная норма (см., например, [1, с. 25]). Как обычно, предполагаем, что $C^{k, 0}(\Gamma)=C^{k}(\Gamma), C^{0, \alpha}(\Gamma)=C^{\alpha}(\Gamma)$ при $\alpha<1$.

Пусть $\zeta(t)$ - диффеоморфизм класса $C^{k, \alpha}(\Gamma), k \geqslant 1,0 \leqslant \alpha \leqslant 1$, контура Г на себя, причем $\zeta_{t}^{\prime}(t) \neq 0$, где $\zeta_{t}^{\prime}=\zeta_{s}^{\prime} \cdot s_{t}^{\prime}=\zeta_{s}^{\prime} \cdot \bar{t}_{s}^{\prime}, t(s)=e^{i s}$.

Следуя [2, с. 33], для функции $\varphi(t)$, определенной на $\Gamma$, введем оператор сдвига $\mathscr{W} \varphi(t)=\varphi(\zeta(t))$. Очевидно, $\mathscr{W}-$ линейный, ограниченный, непрерывно обратимый в $C^{k, \alpha}(\Gamma), k \geqslant 1,0 \leqslant \alpha \leqslant 1$, оператор, причем $\|\mathscr{W}\|_{C(\Gamma)}=1$ (см. [2, с. 33]).

Обозначим через

$$
S \varphi(t)=\frac{1}{\pi i} \int_{\Gamma} \frac{\varphi(\tau)}{\tau-t} d \tau, \quad t \in \Gamma,
$$

одномерный сингулярный (интегральный) оператор.

При изучении дифференциальных свойств «вплоть до края» решений краевых задач со сдвигом для различных эллиптических систем возникает потребность в исследовании свойств суперпозиции $\mathscr{W} S^{-1}-S$.

Основным результатом этой работы является следующее утверждение.

Теорема 1. Если $\zeta(t) \in C^{1, \alpha}(\Gamma), 0<\alpha \leqslant 1, \varphi(t) \in C^{0, \beta}(\Gamma), 0<\beta \leqslant 1, \mu=\alpha+\beta \leqslant 2$, то при $\mu<1 \Psi \varphi(t)=\left(\mathscr{W} S \mathscr{W}^{-1}-S\right) \varphi(t) \in C^{\mu}(\Gamma)$, причем

$$
\|\Psi \varphi(t)\|_{C^{\mu}(\Gamma)} \leqslant \operatorname{const}\|\varphi(t)\|_{C^{0, \beta}(\Gamma)}
$$

где константа зависит лишь от $\|\zeta\|_{C^{1, \alpha}(\Gamma)}$.

(c) 2017 Климентов С. Б. 
Если $\mu=1$, то $\Psi \varphi(t) \in C^{\mu-\varepsilon}(\Gamma)$ для любого $\varepsilon, 0<\varepsilon<\mu, c$ выполнением оценки, аналогичной (2).

Если $\mu>1$, то $\Psi \varphi(t) \in C^{1, \mu-1}(\Gamma)$, причем

$$
\|\Psi \varphi(t)\|_{C^{1, \mu-1}(\Gamma)} \leqslant \mathrm{const}\|\varphi(t)\|_{C^{0, \beta}(\Gamma)}
$$

где константа зависит лишь от $\|\zeta\|_{C^{1, \alpha}(\Gamma)}$.

Очевидно

Следствие 1. Если $\zeta(t) \in C^{1, \alpha}(\Gamma), 0<\alpha \leqslant 1, \varphi(t) \in C^{1, \beta}(\Gamma), 0<\beta \leqslant 1$, то $\Psi \varphi(t) \in$ $C^{1, \alpha}(\Gamma)$, причем

$$
\|\Psi \varphi(t)\|_{C^{1, \alpha}(\Gamma)} \leqslant \mathrm{const}\|\varphi(t)\|_{C^{0,1}(\Gamma)} \leqslant \mathrm{const}\|\varphi(t)\|_{C^{1, \beta}(\Gamma)},
$$

где константа зависит лишь от $\|\zeta\|_{C^{1, \alpha}(\Gamma)}$.

ЗАмечАниЕ 1. Как показывают примеры (см. замечание 2 после доказательства теоремы 1$)$, при $\zeta(t) \in C^{1, \alpha}(\Gamma)$ показатель $\alpha$ в левой части (4) не улучшаем в том смысле, что существуют функции $\zeta(t) \in C^{1, \alpha}(\Gamma), \varphi(t) \in C^{1, \alpha}(\Gamma)$ такие, что $\Psi \varphi(t) \in C^{1, \alpha}(\Gamma)$, но $\Psi \varphi(t) \notin C^{1, \gamma}(\Gamma)$ при любом $\gamma, 1 \geqslant \gamma>\alpha$.

\section{2. Вспомогательные построения}

Положим $t=e^{i s}, \tau=e^{i \sigma}, \omega=r \sigma+(1-r) s, 0 \leqslant r \leqslant 1, u=e^{i \omega}$.

Лемма 1. Если $\zeta(t) \in C^{n+1, \alpha}(\Gamma), n \geqslant 0,0<\alpha \leqslant 1$, то имеют место следующие разновидности формулы Тэйлора:

$$
\begin{gathered}
\zeta(\tau)-\zeta(t)=\zeta^{\prime}(t)(\tau-t)+\zeta^{\prime \prime}(t) \frac{(\tau-t)^{2}}{2 !}+\cdots \\
+\zeta^{(n)}(t) \frac{(\tau-t)^{n}}{n !}+\frac{(\tau-t)^{n+1}}{n !} \int_{0}^{1} \Omega(r, s, \sigma, \omega) \zeta^{(n+1)}\left(e^{i \omega}\right)(1-r)^{n} d r
\end{gathered}
$$

где

$$
\begin{gathered}
\Omega(r, s, \sigma, \omega)=i \cdot\left[\frac{\tau-t}{\sigma-s}\right]^{-n-1} \cdot e^{i(n+1) \omega} \cdot\left[\frac{e^{i(\sigma-s)(1-r)}-1}{(\sigma-s)(1-r)}\right]^{n} ; \\
\zeta(\tau)-\zeta(t)=\zeta^{\prime}(t)(\tau-t)+\zeta^{\prime \prime}(t) \frac{(\tau-t)^{2}}{2 !}+\cdots \\
+\zeta^{(n)}(t) \frac{(\tau-t)^{n}}{n !}+\zeta^{(n+1)}(t) \frac{(\tau-t)^{n+1}}{(n+1) !}+\Omega_{1}(\tau, t),
\end{gathered}
$$

где остаточный член имеет вид

$$
\begin{aligned}
& \Omega_{1}(\tau, t)=\frac{(\tau-t)^{n+1}}{n !} \int_{0}^{1}[\left.\Omega(r, s, \sigma, \omega) \zeta^{(n+1)}\left(e^{i \omega}\right)-\zeta^{(n+1)}(t)\right](1-r)^{n} d r \\
&=O\left(|\tau-t|^{n+1+\alpha}\right) .
\end{aligned}
$$

Здесь запись $f(x)=O(\varphi(x))$ означает выполнение неравенства

$$
|f(x)| \leqslant \operatorname{const}|\varphi(x)|,
$$

где const от $f$ и $\varphi$ не зависит. 
$\triangleleft$ ДОКАЗАТЕЛЬСТВО лЕммЫ 1. Для функции $f(t) \in C^{1}(\Gamma)$ очевидно соотношение:

$$
f(\tau)-f(t)=\int_{t}^{\tau} f^{\prime}(u) d u .
$$

Подставив в (9)

$$
f(u)=\zeta(u)+(\tau-u) \zeta^{\prime}(u)+\frac{(\tau-u)^{2}}{2} \zeta^{\prime \prime}(u) \frac{(\tau-u)^{3}}{3 !} \zeta^{\prime \prime \prime}(u)+\cdots+\frac{(\tau-u)^{n}}{n !} \zeta^{(n)}(u),
$$

в получившемся интеграле перейдем к переменной интегрирования $r$, после чего получим (5).

Легко проверяется соотношение

$$
\Omega(r, s, \sigma, \omega)=1+O(|\tau-t|) .
$$

Действительно,

$$
\begin{gathered}
i \cdot\left[\frac{\tau-t}{\sigma-s}\right]^{-n-1}=e^{-i(n+1) s}\left[\frac{e^{i(\sigma-s)}-1}{\sigma-s}\right]^{-(n+1)} \\
=e^{-i(n+1) s} \cdot i^{-n} \cdot[1+O(\sigma-s)], \\
{\left[\frac{e^{i(\sigma-s)(1-r)}-1}{(\sigma-s)(1-r)}\right]^{n}=i^{n} \cdot[1+O(\sigma-s)],}
\end{gathered}
$$

и

$$
e^{-i(n+1) s} \cdot e^{i(n+1) \omega}=e^{i r(\sigma-s)(n+1)}=1+O(\sigma-s) .
$$

Поскольку

$$
0<\text { const } \leqslant \frac{\left|t_{2}-t_{1}\right|}{\left|s_{2}-s_{1}\right|} \leqslant 1
$$

для любых $t_{1}, t_{2} \in \Gamma\left(s_{1}, s_{2}\right.$ - дуговые абсциссы этих точек), в этих формулах можно заменить $O(\sigma-s)$ на $O(|\tau-t|)$. Проделав такую замену, из (6) получим (10).

Далее, формально записав равенство (7) и вычтя из него (5), с использованием (10) получим (8). Последнее равенство в (8) следует из $(10)$ и $\zeta^{(n+1)}(z) \in C^{\alpha}(\Gamma)$.

\section{3. Доказательство основных результатов}

ДоКАЗАТЕЛЬСТво тЕОРЕмЫ 1. ЗаПишем рассматриваемый оператор следующим образом:

$$
\Psi \varphi(t)=\left(\mathscr{W} S \mathscr{W}^{-1}-S\right) \varphi(t)=\frac{1}{\pi i} \int_{\Gamma} k(\tau, t) \varphi(\tau) d \tau
$$

где

$$
k(\tau, t)=\frac{\zeta^{\prime}(\tau)}{\zeta(\tau)-\zeta(t)}-\frac{1}{\tau-t} .
$$

Поскольку (см. [3, с. 30-31])

$$
\int_{\Gamma} \frac{\zeta^{\prime}(\tau)}{\zeta(\tau)-\zeta(t)} d \tau=\int_{\Gamma} \frac{d \zeta}{\zeta-z}=\pi i, \quad z=\zeta(t) \in \Gamma,
$$


то при $\varphi(\tau)=$ const $\left(\mathscr{W} S \mathscr{W}^{-1}-S\right) \varphi(t) \equiv 0$, т. е.

$$
\int_{\Gamma} k(\tau, t) d \tau \equiv 0
$$

и

$$
\Psi \varphi(t)=\frac{1}{\pi i} \int_{\Gamma} k(\tau, t)\left(\varphi(\tau)-\varphi\left(t_{*}\right)\right) d \tau,
$$

при всяком зафиксированном $t_{*} \in \Gamma$.

Отметим, что (13) можно переписать в виде

$$
k(\tau, t)=\frac{\int_{\tau}^{t}\left[\zeta^{\prime}(u)-\zeta^{\prime}(\tau)\right] d u}{[\zeta(\tau)-\zeta(t)](\tau-t)} .
$$

Зафиксируем на $\Gamma$ точку $t$ и отложим в ту и другую сторону от $t$ дуги $t^{\prime} t$ и $t t^{\prime \prime}$, равные по длине $2 \sigma<\pi$. Обозначим через $l=t^{\prime} t^{\prime \prime}$ объединение этих дуг. Обозначим также через $\lambda, s, \nu$ дуговые абсциссы соответственно точек $\tau, t, w ; w-t=h,|\nu-s|=\sigma$.

Положим в $(15) t_{*}=t$. Тогда будем иметь

$$
\begin{gathered}
\Psi \varphi(t+h)-\Psi \varphi(t) \\
=\frac{1}{\pi i} \int_{\Gamma} k(\tau, t+h)(\varphi(\tau)-\varphi(t+h)) d \tau-\frac{1}{\pi i} \int_{\Gamma} k(\tau, t)(\varphi(\tau)-\varphi(t)) d \tau .
\end{gathered}
$$

Так как $\zeta=\zeta(t)-$ диффеоморфизм, $\left|\zeta^{\prime}(t)\right| \geqslant$ const $>0$, то из $(16)$ и $\zeta(t) \in C^{1, \alpha}(\Gamma)$ получим

$$
|k(\tau, t)| \leqslant \text { const } \cdot \frac{|\tau-t|}{|\zeta(\tau)-\zeta(t)|} \cdot \frac{1}{|\tau-t|^{1-\alpha}} \leqslant \text { const } \cdot \frac{1}{|\tau-t|^{1-\alpha}},
$$

где последняя константа зависит лишь от $\|\zeta\|_{C^{1, \alpha}(\Gamma)}$.

Аналогично

$$
|k(\tau, t+h)| \leqslant \text { const } \cdot \frac{1}{|\tau-t-h|^{1-\alpha}} .
$$

Запишем разность (17) как $I_{0}+I$, где

$$
\begin{aligned}
& I_{0}=\frac{1}{\pi i} \int_{l} k(\tau, t+h)(\varphi(\tau)-\varphi(t+h)) d \tau-\frac{1}{\pi i} \int_{l} k(\tau, t)(\varphi(\tau)-\varphi(t)) d \tau, \\
& I=\frac{1}{\pi i} \int_{\Gamma \backslash l} k(\tau, t+h)(\varphi(\tau)-\varphi(t+h)) d \tau-\frac{1}{\pi i} \int_{\Gamma \backslash l} k(\tau, t)(\varphi(\tau)-\varphi(t)) d \tau .
\end{aligned}
$$

Воспользовавшись $(6)$, из $\varphi(t) \in C^{0, \beta}(\Gamma)$ и (18), (19) выводим

$$
\left|I_{0}\right| \leqslant \operatorname{const}\left\{\int_{s-2 \sigma}^{s+2 \sigma}|\lambda-s-\sigma|^{\mu-1} d \lambda+\int_{s-2 \sigma}^{s+2 \sigma}|\lambda-s|^{\mu-1} d \lambda\right\} \leqslant \text { const }|h|^{\mu},
$$

где $\mu=\alpha+\beta$, а константа зависит от $\|\zeta\|_{C^{1, \alpha}(\Gamma)}$ и линейно от $\|\varphi(t)\|_{C^{0, \beta}(\Gamma)}$. 
Перейдем к оценке выражения $I$, которое запишем в виде

$$
I=I_{1}+I_{2},
$$

где

$$
\begin{gathered}
I_{1}=\frac{1}{\pi i} \int_{\Gamma \backslash l} k(\tau, t)(\varphi(t)-\varphi(t+h)) d \tau, \\
I_{2}=\frac{1}{\pi i} \int_{\Gamma \backslash l}[k(\tau, t+h)-k(\tau, t)](\varphi(\tau)-\varphi(t+h)) d \tau .
\end{gathered}
$$

В силу (14)

$$
I_{1}=\frac{\varphi(t+h)-\varphi(t)}{\pi i} \int_{l} k(\tau, t) d \tau,
$$

и из $(18)$ и $\varphi(t) \in C^{0, \beta}(\Gamma)$ будем иметь

$$
\left|I_{1}\right| \leqslant \text { const }|h|^{\beta} \int_{s-2 \sigma}^{s+2 \sigma} \frac{d \lambda}{|\lambda-s|^{1-\alpha}} \leqslant \text { const }|h|^{\alpha+\beta},
$$

где константа зависит от $\|\zeta\|_{C^{1, \alpha}(\Gamma)}$ и линейно от $\|\varphi\|_{C^{0, \beta}(\Gamma)}$.

Перейдем к оценке выражения $I_{2}$. Для этого преобразуем разность $k(\tau, t+h)-k(\tau, t)$. Ясно, что это будет некоторая дробь $\frac{P(\tau, t, h)}{Q(\tau, t, h)}$, где

$$
Q(\tau, t, h)=[\zeta(\tau)-\zeta(t+h)] \cdot[\zeta(\tau)-\zeta(t)] \cdot(\tau-t) \cdot(\tau-t-h) .
$$

Учитывая $(16)$, для $P(\tau, t, h)$ будем иметь

$$
\begin{aligned}
P(\tau, t, h)= & \left\{\int_{\tau}^{t}\left[\zeta^{\prime}(u)-\zeta^{\prime}(\tau)\right] d u+\int_{t}^{t+h}\left[\zeta^{\prime}(u)-\zeta^{\prime}(\tau)\right] d u\right\}\left\{-\int_{\tau}^{t} \zeta^{\prime}(u) d u\right\}(\tau-t) \\
& -\int_{\tau}^{t}\left[\zeta^{\prime}(u)-\zeta^{\prime}(\tau)\right] d u\left\{-\int_{\tau}^{t} \zeta^{\prime}(u) d u-\int_{t}^{t+h} \zeta^{\prime}(u) d u\right\}(\tau-t) \\
& -h \int_{\tau}^{t}\left[\zeta^{\prime}(u)-\zeta^{\prime}(\tau)\right] d u \int_{\tau}^{t+h} \zeta^{\prime}(u) d u \\
= & \zeta^{\prime}(\tau) \int_{t}^{t+h} \zeta^{\prime}(u) d u(\tau-t)(\tau-t-h)-h \int_{\tau}^{t} \zeta^{\prime}(u) d u \int_{\tau}^{t+h} \zeta^{\prime}(u) d u .
\end{aligned}
$$

Поскольку $\zeta(t) \in C^{1, \alpha}(\Gamma), 0<\alpha \leqslant 1$, имеют место следующие соотношения (см. леммy 1):

$$
\begin{gathered}
\int_{t}^{t+h} \zeta^{\prime}(u) d u=\zeta(t+h)-\zeta(t)=\zeta^{\prime}(t) h+O\left(|h|^{1+\alpha}\right), \\
\int_{\tau}^{t} \zeta^{\prime}(u) d u=\zeta(t)-\zeta(\tau)=-\zeta^{\prime}(t)(\tau-t)+O\left(|\tau-t|^{1+\alpha}\right),
\end{gathered}
$$




$$
\int_{\tau}^{t+h} \zeta^{\prime}(u) d u=-\zeta^{\prime}(\tau)(\tau-t-h)+O\left(|\tau-t-h|^{1+\alpha}\right) .
$$

Подставляя эти соотношения в (25), получим

$$
\begin{gathered}
P(\tau, t, h)=\zeta^{\prime}(\tau)(\tau-t)(\tau-t-h) \cdot O\left(|h|^{1+\alpha}\right) \\
+h \zeta^{\prime}(t)(\tau-t) \cdot O\left(|\tau-t-h|^{1+\alpha}\right) \\
+h \zeta^{\prime}(\tau)(\tau-t-h) \cdot O\left(|\tau-t|^{1+\alpha}\right) .
\end{gathered}
$$

Аналогично (20) из (22), (24) и (26) будем иметь

$$
\begin{array}{r}
\left|I_{2}\right| \leqslant \text { const } \cdot|h|^{1+\alpha} \int_{\Gamma \backslash l} \frac{|d \tau|}{|\tau-t| \cdot|\tau-t-h|^{1-\beta}} \\
+ \text { const } \cdot|h| \int_{\Gamma \backslash l} \frac{|d \tau|}{|\tau-t| \cdot|\tau-t-h|^{1-\alpha-\beta}} \\
+ \text { const } \cdot|h| \int_{\Gamma \backslash l} \frac{|d \tau|}{|\tau-t|^{1-\alpha} \cdot|\tau-t-h|^{1-\beta}} .
\end{array}
$$

Так как на $\Gamma \backslash l$ величина $\frac{\sigma}{\lambda-s}$ не превышает по абсолютной величине $\frac{1}{2}$, из $(27)$ получаем

$$
\begin{aligned}
& \left|I_{2}\right| \leqslant \text { const } \cdot \sigma^{1+\alpha}\left\{\int_{s+2 \sigma}^{\pi+s} \frac{d \lambda}{|\lambda-s|^{2-\beta}}+\int_{-\pi+s}^{s-2 \sigma} \frac{d \lambda}{|\lambda-s|^{2-\beta}}\right\} \\
& + \text { const } \cdot \sigma\left\{\int_{s+2 \sigma}^{\pi+s} \frac{d \lambda}{|\lambda-s|^{2-\alpha-\beta}}+\int_{-\pi+s}^{s-2 \sigma} \frac{d \lambda}{|\lambda-s|^{2-\alpha-\beta}}\right\} .
\end{aligned}
$$

Если $\alpha+\beta<1$, из (28) будем иметь

$$
\begin{gathered}
\left|I_{2}\right| \leqslant \text { const } \cdot \sigma^{1+\alpha} \int_{s+2 \sigma}^{\infty} \frac{d \lambda}{(\lambda-s)^{2-\beta}}+\text { const } \cdot \sigma \int_{s+2 \sigma}^{\infty} \frac{d \lambda}{(\lambda-s)^{2-\alpha-\beta}} \\
\leqslant \text { const } \cdot \sigma^{\alpha+\beta} \leqslant \text { const } \cdot|h|^{\alpha+\beta}
\end{gathered}
$$

где константа зависит лишь от $\|\zeta\|_{C^{1, \alpha}(\Gamma)}$ и линейно от $\|\varphi\|_{C^{\beta}(\Gamma)}$.

Если $\alpha+\beta=1$, то, не переходя к бесконечным пределам в интегралах, из (28) получаем

$$
\left|I_{2}\right| \leqslant \text { const } \cdot|h| \ln \frac{1}{|h|}
$$

где также константа зависит лишь от $\|\zeta\|_{C^{1, \alpha}(\Gamma)}$ и линейно от $\|\varphi\|_{C^{\beta}(\Gamma)}$.

Предположим теперь, что $1<\alpha+\beta<2$. В этом случае из (20), (23)-(26) и (28) получаем, что к пределу

$$
\lim _{h \rightarrow 0} \frac{\Psi(t+h)-\Psi(t)}{h}=\frac{1}{\pi i} \lim _{h \rightarrow 0} \int_{\Gamma} \frac{k(\tau, t+h)-k(\tau, t)}{h}(\varphi(\tau)-\varphi(t)) d \tau
$$


применима теорема Лебега о мажорируемой сходимости, т. е. предел можно подвести под знак интеграла и функция $\Psi(t)$ дифференцируема (на $\Gamma$ ) по $t$, причем

$$
\Psi_{t}^{\prime}(t)=\frac{1}{\pi i} \int_{\Gamma} M(\tau, t)(\varphi(\tau)-\varphi(t)) d \tau
$$

где

$$
M(\tau, t)=\frac{\zeta^{\prime}(t) \zeta^{\prime}(\tau)(\tau-t)^{2}-[\zeta(\tau)-\zeta(t)]^{2}}{[\zeta(\tau)-\zeta(t)]^{2}(\tau-t)^{2}}=\frac{\partial}{\partial t} k(\tau, t),
$$

и интеграл в (31) есть обычный абсолютно сходящийся несобственный интеграл. Действительно, с помощью формул

$$
\begin{aligned}
& \zeta(\tau)-\zeta(t)=\zeta^{\prime}(t)(\tau-t)+O\left(|\tau-t|^{1+\alpha}\right), \\
& \zeta(\tau)-\zeta(t)=\zeta^{\prime}(\tau)(\tau-t)+O\left(|\tau-t|^{1+\alpha}\right)
\end{aligned}
$$

числитель дроби в (32) преобразуется следующим образом:

$$
\begin{gathered}
\zeta^{\prime}(t) \zeta^{\prime}(\tau)(\tau-t)^{2}-[\zeta(\tau)-\zeta(t)]^{2} \\
=\zeta^{\prime}(t)(\tau-t) \cdot O\left(|\tau-t|^{1+\alpha}\right)+\zeta^{\prime}(\tau)(\tau-t) \cdot O\left(|\tau-t|^{1+\alpha}\right),
\end{gathered}
$$

откуда, с учетом $\varphi(t) \in C^{0, \beta}(\Gamma)$, будем иметь

$$
|M(\tau, t)(\varphi(\tau)-\varphi(t))| \leqslant \text { const } \cdot \frac{1}{|\tau-t|^{2-\alpha-\beta}},
$$

где константа зависит лишь от $\|\zeta\|_{C^{1, \alpha}(\Gamma)}$ и линейно от $\|\varphi\|_{C^{0, \beta}(\Gamma)}$.

Используя схему, аналогичную примененной к $\Psi(t)$, покажем, что $\Psi_{t}^{\prime}(t) \in C^{\alpha+\beta-1}(\Gamma)$.

Аналогично (17) запишем

$$
\begin{gathered}
\Psi_{t}^{\prime}(t+h)-\Psi_{t}^{\prime}(t) \\
=\frac{1}{\pi i} \int_{\Gamma} M(\tau, t+h)(\varphi(\tau)-\varphi(t+h)) d \tau-\frac{1}{\pi i} \int_{\Gamma} M(\tau, t)(\varphi(\tau)-\varphi(t)) d \tau
\end{gathered}
$$

и представим эту разность как $J_{0}+J$, где

$$
\begin{aligned}
& J_{0}=\frac{1}{\pi i} \int_{l} M(\tau, t+h)(\varphi(\tau)-\varphi(t+h)) d \tau-\frac{1}{\pi i} \int_{l} M(\tau, t)(\varphi(\tau)-\varphi(t)) d \tau, \\
& J=\frac{1}{\pi i} \int_{\Gamma \backslash l} M(\tau, t+h)(\varphi(\tau)-\varphi(t+h)) d \tau-\frac{1}{\pi i} \int_{\Gamma \backslash l} M(\tau, t)(\varphi(\tau)-\varphi(t)) d \tau
\end{aligned}
$$

(здесь дуга $l$ та же, что и выше).

Аналогично (20), из (34) имеем

$$
\left|J_{0}\right| \leqslant \operatorname{const}\left\{\int_{s-2 \sigma}^{s+2 \sigma}|\lambda-s-\sigma|^{\mu-2} d \lambda+\int_{s-2 \sigma}^{s+2 \sigma}|\lambda-s|^{\mu-2} d \lambda\right\} \leqslant \text { const }|h|^{\mu-1},
$$

где $\mu=\alpha+\beta$. 
Далее, аналогично предыдущему, представим $J$ в виде суммы $J=J_{1}+J_{2}$, где

$$
\begin{gathered}
J_{1}=\frac{1}{\pi i} \int_{\Gamma \backslash l} M(\tau, t)(\varphi(t)-\varphi(t+h)) d \tau, \\
J_{2}=\frac{1}{\pi i} \int_{\Gamma \backslash l}[M(\tau, t+h)-M(\tau, t)](\varphi(\tau)-\varphi(t+h)) d \tau .
\end{gathered}
$$

Аналогично (23) будем иметь

$$
\left|J_{1}\right| \leqslant \mathrm{const}|h|^{\beta} \int_{s-2 \sigma}^{s+2 \sigma} \frac{d \lambda}{|\lambda-s|^{2-\alpha}} \leqslant \text { const }|h|^{\alpha+\beta-1},
$$

где константа зависит лишь от $\|\zeta\|_{C^{1, \alpha}(\Gamma)}$ и линейно от $\|\varphi\|_{C^{0, \beta}(\Gamma)}$.

Далее, для оценки $J_{2}$ преобразуем разность

$$
M(\tau, t+h)-M(\tau, t)=\frac{P_{1}(\tau, t, h)}{Q_{1}(\tau, t, h)},
$$

где

$$
\begin{gathered}
Q_{1}(\tau, t, h)=[\zeta(\tau)-\zeta(t)]^{2}[\zeta(\tau)-\zeta(t+h)]^{2}(\tau-t-h)^{2}(\tau-t)^{2}, \\
P_{1}(\tau, t, h)=\left\{\zeta^{\prime}(t+h) \zeta^{\prime}(\tau)(\tau-t-h)^{2}-[\zeta(\tau)-\zeta(t+h)]^{2}\right\}[\zeta(\tau)-\zeta(t)]^{2}(\tau-t)^{2} \\
-\left\{\zeta^{\prime}(t) \zeta^{\prime}(\tau)(\tau-t)^{2}-[\zeta(\tau)-\zeta(t)]^{2}\right\}[\zeta(\tau)-\zeta(t+h)]^{2}(\tau-t-h)^{2},
\end{gathered}
$$

или, после элементарных преобразований,

$$
\begin{gathered}
P_{1}(\tau, t, h)=\zeta^{\prime}(t+h) \zeta^{\prime}(\tau)(\tau-t-h)^{2}[\zeta(\tau)-\zeta(t)]^{2}(\tau-t)^{2} \\
-[\zeta(\tau)-\zeta(t+h)]^{2}[\zeta(\tau)-\zeta(t)]^{2}(\tau-t)^{2} \\
-\zeta^{\prime}(t) \zeta^{\prime}(\tau)(\tau-t)^{2}[\zeta(\tau)-\zeta(t+h)]^{2}(\tau-t-h)^{2} \\
+[\zeta(\tau)-\zeta(t)]^{2}[\zeta(\tau)-\zeta(t+h)]^{2}(\tau-t-h)^{2} \\
=\zeta^{\prime}(\tau)(\tau-t)^{2}(\tau-t-h)^{2}\left\{\left[\zeta^{\prime}(t+h)-\zeta^{\prime}(t)\right][\zeta(\tau)-\zeta(t)]^{2}\right. \\
\left.+\zeta^{\prime}(t)\left[(\zeta(\tau)-\zeta(t))^{2}-(\zeta(\tau)-\zeta(t)+\zeta(t)-\zeta(t+h))^{2}\right]\right\} \\
+[\zeta(\tau)-\zeta(t)]^{2}[\zeta(\tau)-\zeta(t+h)]^{2}\left[(\tau-t-h)^{2}-(\tau-t)^{2}\right] \\
=\zeta^{\prime}(\tau)(\tau-t)^{2}(\tau-t-h)^{2}\left\{\left[\zeta^{\prime}(t+h)-\zeta^{\prime}(t)\right][\zeta(\tau)-\zeta(t)]^{2}\right. \\
\left.-\zeta^{\prime}(t)\left[2(\zeta(\tau)-\zeta(t))(\zeta(t)-\zeta(t+h))+(\zeta(t)-\zeta(t+h))^{2}\right]\right\} \\
+[\zeta(\tau)-\zeta(t)]^{2}[\zeta(\tau)-\zeta(t+h)]^{2}\left[-2 h(\tau-t)+h^{2}(\tau-t)^{2}\right]
\end{gathered}
$$

Используя (33), а также формулу

$$
\zeta(t)-\zeta(t+h)=-\zeta^{\prime}(t) h+O\left(|h|^{1+\alpha}\right),
$$

из $(41)$ для $P_{1}(\tau, t, h)$ получим следующее выражение:

$$
\begin{gathered}
P_{1}(\tau, t, h)=h^{\alpha} \cdot O\left(|\tau-t|^{4}|\tau-t-h|^{2}\right) \\
+h \cdot\left[O\left(|\tau-t|^{3+\alpha}|\tau-t-h|^{2}\right)+O\left(|\tau-t|^{2+\alpha}|\tau-t-h|^{3}\right)\right] \\
+h^{1+\alpha} \cdot O\left(|\tau-t|^{3}|\tau-t-h|^{2}\right) .
\end{gathered}
$$


Поскольку на $\Gamma \backslash l$ величина $\frac{\sigma}{\lambda-s}$ не превышает по абсолютной величине $\frac{1}{2}$, из $(40)$, (43), на $\Gamma \backslash l$ имеем оценку

$$
\begin{gathered}
\mid(M(\tau, t+h)-M(\tau, t)(\varphi(\tau)-\varphi(t+h) \mid \\
\leqslant \text { const }\left\{\frac{\sigma^{\alpha}}{|\lambda-s|^{2-\beta}}+\frac{\sigma}{|\lambda-s|^{3-\mu}}+\frac{\sigma^{1+\alpha}}{|\lambda-s|^{3-\beta}}\right\},
\end{gathered}
$$

где, как и выше, $t=e^{i s}, \tau=e^{i \lambda}, \mu=\alpha+\beta$, а константа зависит лишь от $\|\zeta\|_{C^{1, \alpha}(\Gamma)}$ и линейно от $\|\varphi\|_{C^{0, \beta}(\Gamma)}$.

Аналогично (28), (29), из (44) получим

$$
\left|J_{2}\right| \leqslant \text { const } \cdot|h|^{\alpha+\beta-1} .
$$

Сопоставляя (20), (23), (29), (30), (36), (39) и (45), получаем утверждение теоремы 1. $\triangleright$

Непосредственно из рассуждений доказательства теоремы 1 вытекает

Следствие 2. Если $\zeta(z) \in C^{1, \alpha}(\bar{D})$ - голоморфное продолжение $\zeta(t)$ внутрь $D$, а $\Psi(z)$ определяется формулами (12), (13) с заменой переменной $t$ на $z$, то

$$
\lim _{z \rightarrow t} \Psi(z)=\Psi(t), \quad \lim _{z \rightarrow t} \frac{\partial}{\partial z} \Psi(z)=\Psi^{\prime}(t), \quad z \in D, t \in \Gamma .
$$

ЗАмечАниЕ 2. Обсудим несколько подробнее банахово пространство (комплекснозначных) функций $C^{1, \alpha}(\Gamma)$. Норма в нем, как известно $[1$, с. 25$]$, задается формулой

$$
\|\varphi(t)\|_{C^{1, \alpha}(\Gamma)}=\max _{t \in \Gamma}|\varphi(t)|+\max _{t \in \Gamma}\left|\varphi^{\prime}(t)\right|+\sup _{\tau, t \in \Gamma} \frac{\left|\varphi^{\prime}(\tau)-\varphi^{\prime}(t)\right|}{|\tau-t|^{\alpha}} .
$$

Обозначим через $C_{0}^{1, \alpha}(\Gamma)$ подпространство функций $\varphi(t) \in C^{1, \alpha}(\Gamma)$, для которых

$$
\lim _{|\tau-t| \rightarrow 0} \frac{\left|\varphi^{\prime}(\tau)-\varphi^{\prime}(t)\right|}{|\tau-t|^{\alpha}}=0
$$

$\left(C_{0}^{1, \alpha}(\Gamma)\right.$ - замкнутое подпространство пространства $C^{1, \alpha}(\Gamma)$ (см. [4, с. 269]).)

Обозначим $C_{*}^{1, \alpha}(\Gamma)=C^{1, \alpha}(\Gamma) \backslash C_{0}^{1, \alpha}(\Gamma), D \subset C^{1, \alpha}(\Gamma)$ - множество диффеоморфизмов окружности $\Gamma$. Покажем, что $D_{*}=D \cap C_{*}^{1, \alpha}(\Gamma) \neq \varnothing$.

Пусть $0<\alpha<1$. Положим

$$
\varphi(s)= \begin{cases}1, & s \in[0, \pi-2], \\ \frac{1}{2}\left(1+|s-\pi+1|^{\alpha}\right), & s \in[\pi-2, \pi], \\ \frac{1}{2}\left(3-|s-\pi-1|^{\alpha}\right), & s \in[\pi, \pi+2], \\ 1, & s \in[\pi+2,2 \pi]\end{cases}
$$

и

$$
\varphi^{*}(s)= \begin{cases}1, & s \in[0, \pi-2], \\ \frac{1}{2}\left(3-|s-\pi+1|^{\alpha}\right), & s \in[\pi-2, \pi], \\ \frac{1}{2}\left(1+|s-\pi-1|^{\alpha}\right), & s \in[\pi, \pi+2], \\ 1, & s \in[\pi+2,2 \pi],\end{cases}
$$


а также

$$
f(s)=\int_{0}^{s} \varphi(\sigma) d \sigma, \quad f^{*}(s)=\int_{0}^{s} \varphi^{*}(\sigma) d \sigma, \quad s \in[0,2 \pi] .
$$

Очевидно, функции $\zeta(t)=\zeta\left(e^{i s}\right)=\zeta(s)=e^{i f(s)}$ и $\zeta^{*}(t)=e^{i f^{*}(s)}$ задают диффеоморфизмы класса $C^{1, \alpha}(\Gamma)$ окружности $Г$ на себя и $\zeta(t), \zeta^{*}(t) \in C_{*}^{1, \alpha}(\Gamma)$.

Отметим, что

$$
\zeta^{*}(t)=\overline{\zeta(t)} \cdot t^{2}
$$

Введем в рассмотрение два линейных оператора

$$
P_{+}=\frac{1}{2}(I+S), \quad P_{-}=\frac{1}{2}(I-S),
$$

где $I$ - тождественный оператор. Это непрерывные проекторы в прострастве $C^{1, \alpha}(\Gamma)$ (см. [1, с. 38], [3, с. 66]). Для краткости далее будем обозначать проекции $P_{ \pm} C^{1, \alpha}(\Gamma)$ через $P_{ \pm}$.

Ясно, что пространство $C^{1, \alpha}(\Gamma)$ представимо в виде прямой суммы $P_{+} \oplus P_{-}$. На $P_{+}$и $P_{-}$естественным образом определена норма $(47)$ и определенная на $P_{+} \oplus P_{-}$топология произведения $P_{+} \times P_{-}$совпадает с топологией на $C^{1, \alpha}(\Gamma)$, определенной нормой $(47)$.

Рассмотрим разложение в ряд Фурье функции $\zeta(t)=\zeta\left(e^{i s}\right)$ :

$$
\zeta\left(e^{i s}\right)=\sum_{n=1}^{\infty}\left[c_{0}+c_{-n} e^{-i n s}+c_{n} e^{i n s}\right] .
$$

Очевидно, $P_{-} \zeta\left(e^{i s}\right)=\sum_{n=1}^{\infty} c_{-n} e^{-i n s}$, а $P_{+} \zeta\left(e^{i s}\right)=\sum_{n=1}^{\infty}\left[c_{0}+c_{n} e^{i n s}\right]$.

Продифференцировав ряд (49) по $s$, из теоремы 4.7 и формулы $(4.1)$ из [5, с. 79, 81], получим следующее утверждение.

Лемма 2. Если $\zeta(t) \in C^{1, \alpha}(\Gamma), 0<\alpha \leqslant 1$, то $c_{\nu}=O\left(\frac{1}{|\nu|^{1+\alpha}}\right)$. Для того чтобы $\zeta(t) \in C_{*}^{1, \alpha}$, необходимо и достаточно, чтобы нашлись коэффициенты $c_{\nu}$ разложения (49) со сколь угодно большими по модулю номерами такие, что $\left|c_{\nu}\right| \geqslant \frac{L}{\left.\nu\right|^{1+\alpha}}$, где константа $L>0$ от $\nu$ не зависит.

$\triangleleft$ Пусть $\zeta(t) \in C_{*}^{1, \alpha}(\Gamma)-$ построенный выше диффеоморфизм окружности Г. Положим в теореме $1 \varphi(\tau)=\zeta(\tau)$. Тогда

$$
\Psi \zeta(t)=-\frac{1}{\pi i} \int_{\Gamma} \frac{\zeta(\tau)}{\tau-t} d \tau+\zeta(t)=2 P_{-} \zeta(t) . \triangleright
$$

Если $P_{-} \zeta(t) \notin C_{*}^{1, \alpha}(\Gamma)$, то в силу леммы 2 и $(48) P_{-} \zeta^{*}(t) \in C_{*}^{1, \alpha}(\Gamma)$ и в качестве $\varphi(\tau)$ возьмем $\zeta^{*}(t)$. Таким образом, можем считать, что $\Psi \zeta(t) \in C_{*}^{1, \alpha}(\Gamma)$, откуда $\Psi \zeta(t) \notin$ $C^{1, \gamma}(\Gamma)$ для любого $\gamma, 1 \geqslant \gamma>\alpha$.

\section{Литература}

1. Векуа И. Н. Обобщенные аналитические функции.-М.: Физматгиз, 1959.-628 с.

2. Литвинчук Г. С. Краевые задачи и сингулярные интегральные уравнения со сдвигом.-М.: Физматгиз, 1977.-448 с.

3. Гахов Ф. Д. Краевые задачи.-М.: Наука, 1977.-640 с. 
4. Крейн С. Г., Петунин Ю. И., Семенов Е. М. Интерполяция линейных операторов.-М.: Наука, 1978. -400 c.

5. Зигмунд А. Тригонометрические ряды. Т. 1.-М.: Мир, 1965.-615 с.

Статъл поступила 25 октлбря 2016 г.

КлИМЕНТОв СЕРГЕЙ БОРИСОВич

Южный математический институт - филиал ВНЦ РАН,

ведущий научный сотрудник отдела математического анализа

РОССИЯ, 362027, Владикавказ, ул. Маркуса, 22;

Южный федеральный университет,

заведующий кафедрой геометрии

РОССИЯ, 344090, Ростов-на-Дону, ул. Мильчакова, 8 а

E-mail: sklimentov@hotmail.com

\section{ON COMBINATIONS OF THE CIRCLE SHIFTS \\ AND SOME ONE-DIMENSIONAL INTEGRAL OPERATORS}

Klimentov S. B.

The diffeomorphism $\zeta=\zeta\left(e^{i s}\right)$ of the unit circle and the operator $\Psi \varphi(t)=\frac{1}{\pi i} \int_{\Gamma}\left[\frac{\zeta^{\prime}(\tau)}{\zeta(\tau)-\zeta(t)}-\frac{1}{\tau-t}\right] \varphi(\tau) d \tau$ are under consideration. The main results can be stated as follows: If $\zeta(t) \in C^{1, \alpha}(\Gamma), 0<\alpha \leqslant 1$, $\varphi(t) \in C^{0, \beta}(\Gamma), 0<\beta \leqslant 1, \mu=\alpha+\beta \leqslant 2$, then $\Psi \varphi(t) \in C^{\mu}(\Gamma)$ for $\mu<1$. Moreover, the following inequality holds:

$$
\|\Psi \varphi(t)\|_{C^{\mu}(\Gamma)} \leqslant \mathrm{const}\|\varphi(t)\|_{C^{0, \beta}(\Gamma)},
$$

where the constant depends on $\|\zeta\|_{C^{1, \alpha}(\Gamma)}$ only. If $\mu=1$, then $\Psi \varphi(t) \in C^{\mu-\varepsilon}(\Gamma)$ for all $0<\varepsilon<\mu$ and the similar inequality holds. If $\mu>1$, then $\Psi \varphi(t) \in C^{1, \mu-1}(\Gamma)$, and

$$
\|\Psi \varphi(t)\|_{C^{1, \mu-1}(\Gamma)} \leqslant \mathrm{const}\|\varphi(t)\|_{C^{0, \beta}(\Gamma)},
$$

where the constant depends on $\|\zeta\|_{C^{1, \alpha}(\Gamma)}$ only. If $\zeta(t) \in C^{1, \alpha}(\Gamma), 0<\alpha \leqslant 1, \varphi(t) \in C^{1, \beta}(\Gamma), 0<\beta \leqslant 1$, then $\Psi \varphi(t) \in C^{1, \alpha}(\Gamma)$, and

$$
\|\Psi \varphi(t)\|_{C^{1, \alpha}(\Gamma)} \leqslant \text { const }\|\varphi(t)\|_{C^{0,1}(\Gamma)} \leqslant \text { const }\|\varphi(t)\|_{C^{1, \beta}(\Gamma)},
$$

where the constant depends on $\|\zeta\|_{C^{1, \alpha}(\Gamma)}$ only. The index $\alpha$ in the left-hand side of the last inequality can not be improved. The appropriate example is given.

Key words: shift, singular integral operator. 